\title{
Potential Models of Group Learning in Production
}

\author{
Jaakko PELTOKORPI $^{\mathrm{a}, 1}$ and Mohamad Y. JABER ${ }^{\mathrm{b}}$ \\ ${ }^{a}$ Department of Mechanical Engineering, Aalto University School of Engineering, \\ Puumiehenkuja 3, 02150, Espoo, Finland \\ ${ }^{\mathrm{b}}$ Department of Mechanical and Industrial Engineering, Ryerson University, \\ 350 Victoria Street, Toronto, Ontario, M5B 2K3, Canada
}

\begin{abstract}
Working in groups is beneficial for many complex production jobs as groups can have the cognitive and physical capacity that lacks from individuals. The group learning process is complicated when, in addition to individual learning by doing, the number of workers and knowledge transfer have their effects. Production managers need tools for analyzing and predicting group performance and learning over future production periods. Mathematical learning curve models are one of those tools that managers use, with a few are available for groups. This paper reviews potential group learning curve models for production environments. The models are fitted to data from an assembly experiment consisting of different group sizes and repetitions. The results show that more parameters improve the fit. A qualitative evaluation has been performed to answer how well the models reflect group learning and support decision making in production and how their prediction of data could be improved. The results suggest that the S-shaped model performed the best making it a potential one for describing learning in groups in production environments. The paper also suggests future directions along with this line of research.
\end{abstract}

Keywords. Group learning, learning curve model, group size, production, experimental data

\section{Introduction}

Learning in production can be defined as the acquisition of knowledge or skills through direct experience of carrying out a task [1]. In the learning curve literature, the number of task repetitions, or cumulative production, has commonly been used as a proxy to measuring experience [2,3]. Wright [4] was the first to observe the learning phenomenon in a manufacturing setting and to develop a mathematical model to describe the relationship between performance (time/cost per unit) and output (cumulative production). He found that performance improves each time the output doubles. His simple power-form (also referred to as the log-linear) model has been a good starting point for developing new learning curve models that are better at representing learning data in different production environments.

Besides individual learning, group learning has received growing attention. Production of complex products often requires a group of several workers for cognitively

\footnotetext{
${ }^{1}$ Corresponding Author. jaakko.peltokorpi@aalto.fi
} 
and physically demanding tasks. On the other hand, parallel subtasks and the workspace the production of a product needs may justify for managers forming working groups. Lot production is typical in manufacturing. Frequent product changes require groups to adapt continually and, thus, learn new tasks. However, the learning processes of individuals and, in particular, those of groups are complicated. Simple enough mathematical models would help in predicting or analyzing group learning in future production periods. Previous literature offers only a few specific group learning curve models (e.g., [5-8]). Grosse et al. [9] reviewed models and fitted them to group learning data. Their data was not for a production task, nor it considered varying group sizes. This paper addresses this gap in the literature and reviews learning models that could mathematically describe group learning in production. One of the aspects this study focuses on is modeling knowledge transfer as group learning is dependent on interaction and communication among workers as well as the group size. The knowledge transfer curve is affected by two variables: content (e.g., quality and quantity of information) and speed (the time to transfer and realize knowledge) [10]. This paper reviews potential group learning models (Section 1) and their fit to learning data from the experimental assembly study (Section 2). Models are also qualitatively evaluated (Section 3) to see how well they reflect group learning and support decision making in production. The paper concludes with some remarks and suggested future research directions (Section 4).

\section{Learning curve models}

This section reviews potential mathematical learning curve models that could describe group learning in production. It also divides them into univariate and bivariate models. The first group has cumulative production alone as the independent variable, while the second group has an additional variable, which is the number of workers in a group.

\subsection{Univariate models}

The first and most commonly used mathematical expression of learning is the powerform model by Wright [4]:

$$
y_{x}=y_{1} x^{-b}
$$

where $y_{x}$ and $y_{1}$ are unit times to perform the $x$ th and the first repetitions, respectively, $b=\log \phi / \log 2$, with $0 \leq b<1$, is the learning exponent, and $\phi$ is learning rate. The unit time in Eq. (1) decreases at a constant rate with diminishing returns. The Wright's learning curve, WLC, is an aggregation of the learning curves of many workers and does not account for the number of workers. A drawback of Eq. (1) is that as the number of repetitions approaches a large number, the time per unit approaches zero, which is unrealistic, which was corrected in a plateau model:

$$
y_{x}=\left(y_{1}-c\right) x^{-b}+c
$$

where two components are assumed, one subject to improvement, similar to WLC, and the other, $c$, subject to no improvement. Peltokorpi and Niemi [11] found that the de Jong model [12], similar to Eq. (2), fitted group learning data very well. It is worth noting 
that they did not set an upper limit for $b$, which this study does, $0 \leq b<1$, to ensure a fair comparison with WLC and other models.

The learning curve can also have an exponential form, one of the most common of which is the 2-parameter exponential (2PE) model [13]:

$$
P_{x}=k\left(1-e^{-x / \tau}\right)
$$

where $P_{x}$ is production rate at repetition $x, k$ is the performance asymptote (or maximum performance), and $\tau$ is a learning rate parameter representing how fast $k$ is reached. Learning over time can also be illustrated through a logistic curve of the form:

$$
P_{x}=\frac{L}{1+e^{-s\left(x-x_{0}\right)}}
$$

where $L$ is curve's maximum value (or maximum performance), $s$ is logistic growth rate or steepness of the curve, and $x_{0}$ is cumulative production at the mid-point of the curve.

The logistic curve is S-shaped, reflecting three behaviors of performance. Slow at the start of the curve when a worker or a group is getting familiarized with the task they are performing, steadily improving in the middle and slows again when reaching proficiency [14]. In the social learning literature, e.g., [15], the S-shaped diffusion curve has been extensively used to infer social transfer in both humans and animals. The diffusion curve shows the proportion of individuals that have adopted the information over time. Knowledge transfer requires both transmitters and receivers. When either is rare, such as early or late in the diffusion, the rate of spread is constrained, with the middle one occurring more rapidly than the other two.

\subsection{Bivariate models}

At its simplest, a learning curve model for a group of $n$ workers is obtained by aggregating WLC of each individual, $i=1 \ldots n$, representing a fraction contribution to the production rate, and is hence AWLC, as follows:

$$
P_{x}=\sum_{i=1}^{n} P_{1, i} x^{b_{i}}
$$

Eq. (5) is similar to the product learning curve by Yelle [16], where there are $n$ separate operations to build up a product.

Camm and Womer [5] presented a bivariate group learning curve model, henceforth $\mathrm{CWLC}$, in the context of workforce allocation in aircraft production:

$$
P_{x j}=B n_{x j}^{1 / g} x^{\gamma}
$$

where $P_{x j}$ is production rate for repetition $x$ by group $j$, and $n_{x j}$ size of group $j$ assigned to repetition $x$. Parameter $g$ describes the contribution of the group size, and $\gamma$ is learning exponent. When $n=1$, Eq. (6) equals to WLC. Camm and Womer borrowed the parameters from [17] and estimated the model but did not fit it to data. They assumed that $\gamma>0$ and $g>1$, i.e., production rate increases as a function of both the number of repetitions and group size, the latter with diminishing returns. A recent laboratory study 
[11] confirmed these assumptions. It is worth noting that the model in Eq. (6) does not limit group size to a fixed maximum. Steiner [18] hypothesized that for divisible tasks, there is a limit on group size beyond which productivity decreases with each additional worker, which happens when the contribution of an additional worker is not sufficient to offset the process loss it causes. This behavior is evident in project management [19]; however, no empirical studies have validated such an upper limit in group size at a single production task.

Peltokorpi and Jaber [8] studied a learning curve (PJLC) similar to Eq. (6):

$$
y_{x}=y n^{\alpha} x^{\beta}
$$

where $\alpha$ and $\beta$ are parameters describing the contribution of group size and repetition. They further developed Eq. (7) to an aggregate form by considering different elements in assembly work: $y_{x}=y_{x}^{M}+y_{x}^{C}+y_{x}^{W}=\sum_{i} \gamma_{i} n^{\alpha_{i}} x^{\beta_{i}}$ where $y_{x}^{M}, y_{x}^{C}$ and $y_{x}^{W}$ are time per unit for the motor, cognitive and waste elements of repetition $x$ by group $j$. They fitted the models to assembly learning data from Peltokorpi and Niemi $[11,20]$. This paper will later use the same data to study group learning models. The results from fitting the data provided group size and learning parameter values as follows:

Total assembly time

Motor time

Cognitive time

$$
\begin{aligned}
& y=41.73 n^{-0.717} x^{-0.656} \\
& y=25.179 n^{-0.885} x^{-0.259} \\
& y=8.795 n^{-0.560} x^{-1.949} \\
& y=7.096 n^{-0.209} x^{-1.592}
\end{aligned}
$$

Noteworthy is that the performance of the groups did not exceed the combined effect of individuals, i.e., $\alpha>-1$.

Glock and Jaber [6] developed a learning curve (GJLC) model that aggregates each individual, $i=1, \ldots, n$ learning by doing (similar to AWLC in Eq. 5) and knowledge transfer (similar to 2PE model in Eq. 3) between each pair of individuals:

$$
P_{x}=\sum_{i=1}^{n} P_{1, i} x^{b_{i}}+\sum_{i=1}^{n} \sum_{j=1}^{n} P_{1, j} x^{b_{j}}\left(1-e^{-x / \tau_{i}}\right)
$$

The GJLC model assumes that each unit of knowledge acquired at an individual $j$ through learning by doing may directly be transferred to individual $i$. In Eq. (8), $\tau_{i}=$ $e^{\delta_{i} n}$. Glock and Jaber defined $0<\delta_{i}<2$ as the time delay in the knowledge transfer as a result of increasing group size, $n$. For example, if $\delta_{i}=0.5$, then, for groups of 2 and 4 members, it takes 4.4 and 11.9 repetitions until $80 \%$ of the knowledge available at individual $j$ at the end of repetition $x$ has been transferred to individual $i$. Besides group size, the GJLC model assumes that compatibility of knowledge and willingness to share and absorb knowledge affect its transfer between group members. GJLC assumes a linear relationship for the impact of these parameters that are omitted from Eq. (8) for the sake of simplicity.

The GJLC model fitted better (produced lower MSE) than WLC and AWLC models for 16 group learning datasets [6], 14 of which involved five members and were from the communication experiment in [21] or its extension. The rest two data sets from [22] involved three members in a group. In another study [9], the GJLC model fitted better (higher $\mathrm{R}^{2}$ ) than the 2PE model for 8 out of 12 group learning datasets above. The study [9] concluded that further research should concentrate on modeling group learning in specific industries, and emphasize modeling transfer of knowledge in groups. 
The remaining three learning curve models have productivity of each individual $i$ in a group as dependent variable. Nembhard and Bentefouet [23] extended the 3-parameter hyperbolic model [13] by modeling knowledge transfer from other workers. We omit prior experience to ensure a fair comparison with other models. Their model (NBLC) is as follows:

$$
p_{x, i}=k_{i}\left(\frac{x+\theta_{i} T}{x+\tau_{i}+\theta_{i} T}\right)
$$

where $\theta_{i}$ is percentage of cumulative knowledge, $T=x(n-1)$, that is transferred from other workers. Méndez-Vázquez [7] further extended Nembhard and Bentefouet [23] model by differentiating the effects of knowledge transfer and process loss. She developed both hyperbolic $(\mathrm{H})$ and exponential (E) models, henceforth referred to as MVLC-H and MVLC-E, as follows:

$$
\begin{aligned}
& p_{x, i}=\left(1-L_{n}\right) \varepsilon k_{i}\left(\frac{x+\theta_{i} T}{x+\tau_{i}+\theta_{i} T}\right) \\
& p_{x, i}=\left(1-\varepsilon L_{n}\right) k_{i}\left(1-e^{\frac{-\left(x+\theta_{i} T\right)}{\tau_{i}}}\right)
\end{aligned}
$$

Using the data from Peltokorpi and Niemi [11], the process loss parameter $L_{n}=$ $-0.075+0.088 n$, of the model of Méndez-Vázquez [7], was estimated, which is used later in this paper. The degree of process loss assumes a fixed value $\varepsilon=1$ for each group size. It is worth noting that Eqs. (9)-(11) were used for simulation and optimization purposes. The degrees of knowledge transfer, $\theta$, and process loss, $\varepsilon$, were selected from $0, \ldots, 1$, where zero indicates the worst scenario of knowledge transfer and the best scenario of process loss (i.e., both do not occur at all), and 1 vice versa. We will use $\theta$ as fitting parameter.

\section{Fits}

The ability of learning models, in Eqs. (1)-(11), to produce good fits is tested against data from an experimental study by Peltokorpi and Niemi [11]. In the experiment, students, surrogates to industrial workers, in groups of one to four, assembled a near-real product four times. Groups divided tasks and coordinated work among their members, resembling self-directed worker groups in production. The studied models are divided into three groups based on the dependent variable: Group A (WLC, Plateau and PJLC) has $y_{x}=$ assembly time at repetition $\mathrm{x}$, Group B (2PE, Logistic, AWLC, CWLC and GJLC) $P_{x}=$ group productivity at repetition $\mathrm{x}=1 /$ assembly time at repetition $\mathrm{x}$, Group $\mathrm{C}$ (NBLC, MVLC-H, and MVLC-E) $p_{x}=$ individual productivity at repetition $\mathrm{x}=1 /$ assembly time at repetition $\mathrm{x} /$ group size, $\mathrm{n}$. The parameter values that produced the highest coefficient of determination, $\mathrm{R}^{2}$, and corresponding mean squared error, MSE, for each model within each group size (workers, W), are presented in Table 1. The results show that for one- and four-worker groups, the Logistic model in Group B produces the highest $\mathrm{R}^{2}$, for two-worker groups the 2PE in Group B and the MVLC-E in Group C, and for three-worker groups the Plateau model in Group A (see bolded values). The 
Logistic and Plateau models have three parameters that improve their ability to predict observed data, whereas the WLC and 2PE have two (see Figure 1). The exponential version of MVLC fits better than the hyperbolic one (both have three fitting parameters). MVLC-H and NBLC fit equally. The results also show that the two group learning models, which are the PJLC and the CWLC, appear to fit the entire data set relatively well.

Table 1. Results from fitting the models to the data. The italic values are averages and bolded values represent the best fit.

GROUP A

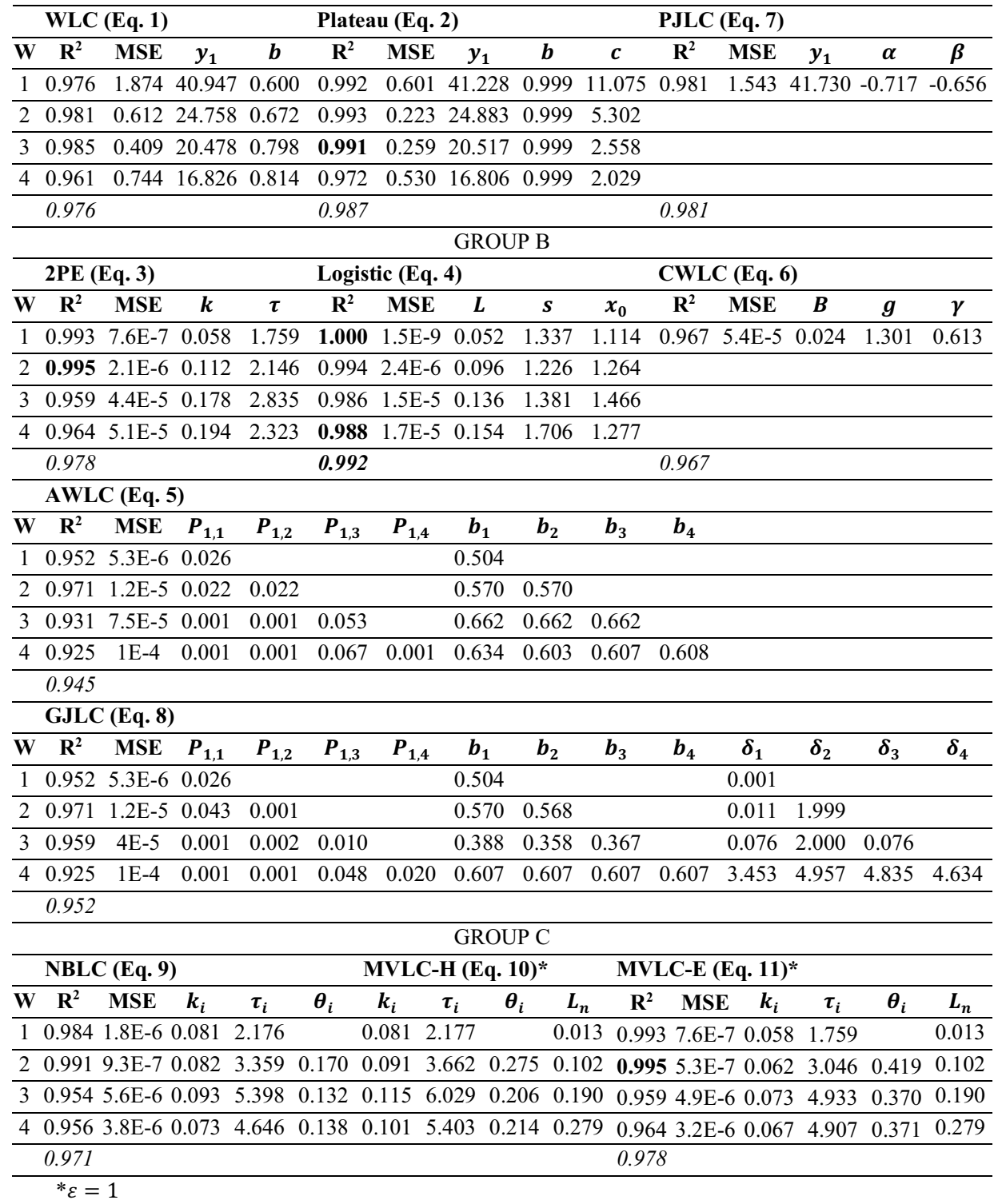




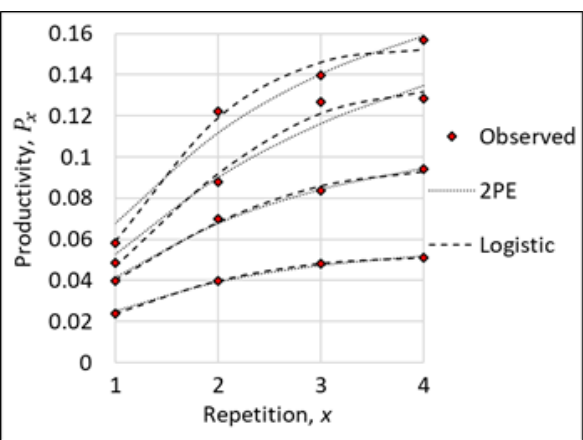

(a)

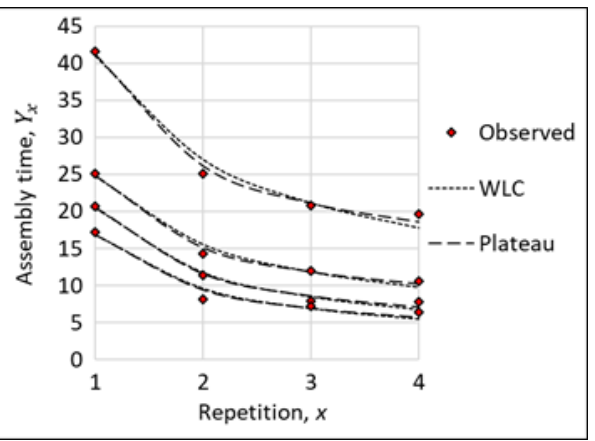

(b)

Figure1. Observed data and predicted learning curves of a) 2PE and Logistic; b) WLC and Plateau.

\section{Evaluation of models for group learning in assembly production}

Although a learning curve model must have the ability to (quantitatively) predict performance in future production periods from the available data, it must also be useful as a decision-making or a continuous improvement measurement tool. In this regard, this section evaluates the parameter values of the models by answering the following questions: How well they reflect group learning in the case assembly and, consequently, in a production environment? What information do they provide to improve performance? What are the limitations of the models and the circumstances under which they could perform the best?

\subsection{The models with cumulative production}

\subsubsection{WLC (Eq. 1)}

The Wright [4] power-form learning curve has two parameters. The first parameter, time to assemble the first unit, $y_{1}$, decreases as a function of group size (Table 1), providing a reasonably accurate estimate of the initial performance and the contribution of adding a worker to a group. The second parameter, the learning exponent, $b$, describing the learning speed, becomes faster with a larger group size, which is an average held over the entire learning period for a group of workers. In this regard, the WLC underestimates the learning speed at the beginning of a learning session and overestimates it towards its end (Figure 1b). Another drawback is that the WLC does not capture plateauing in learning, which, in real situations, occurs for many reasons, such as work organization or quality problems. The above reasons limit the applicability of this model to group learning. It could offer rough performance estimates in production, where, for example, the number of workers varies, and detailed production data is not available.

\subsubsection{Plateau (Eq. 2)}

The Plateau model modifies the WLC model by adding a parameter, $c$, describing the part of the assembly time that is not subject to improvement. As repetitions increase, the learning curve approaches $c$, the standard time for an experienced worker or a group. On the other hand, it could indicate a learning barrier that obstructs further improvements 
unless it is broken. The Plateau model could be suitable for non-standardized production to improve, e.g., work organization and methods. Even if the Plateau model fitted better than WLC for each group size, the learning speeds are in their upper limit, $b=0.999$, and consequently, the $c$ values are unrealistically low (Table 1). Thus, the Plateau model, with a limited learning speed, does not provide reliable estimates when there is considerably rapid learning, such as in the case assembly. Generally speaking, the Plateau model could be a potential model to use instead of the WLC.

\subsection{3. $2 P E(E q .3)$}

The 2-parameter exponential model has a performance asymptote, $k$, which limits learning at a certain level, similar to the plateauing effect. The second parameter, $\tau$, is a learning rate, indicating how fast this level is approached. From Table 1 and Figure 1(a), we can see that larger groups reach higher productivity faster. The $2 \mathrm{PE}$ model could be used to estimate the relative speed for groups of different sizes to reach adequate experience level. On the other hand, the plateau level, $k$ (2PE predicts well for each group size), may indicate learning barriers, and that process should be further improved.

\subsubsection{Logistic (Eq. 4)}

The Logistic model has three parameters. The first parameter, $L$, is the performance limit on a curve, similar to $c$ and $k$ of the Plateau and 2PE models, respectively. The values of $L$, in Table 1, increase as a function of group size, and are lower and, probably, a little less realistic than those of $k$. The second parameter describes the steepness of the curve, $s$, similar to $\tau$ with 2PE. The third parameter, the cumulative production at the mid-point of the curve, $x_{0}$, decreases, on average, as a function of group size. This is the point in the curve where group learning speed is at maximum (note that, in this context, the Sshaped curve, theoretically, starts from $x<0$ ). One can observe learning (which is partly affected by knowledge spread in the group) is relatively slow for larger groups at the first repetition and the end of the learning process, but faster in the middle. This observation suggests that managers should search for and eliminate the barriers that slow learning at both ends of the learning process, especially for larger groups. Often this has to do with work organization, such as division of job and group coordination, which become more complicated with increasing group size.

\subsection{The models with cumulative production and the number of workers}

\subsection{1. $A W L C$ (Eq. 5)}

This model aggregates the fraction contribution of each individual to a group product. Table 1 shows that within each group size, workers are similar learners (equal learning exponents), but within groups of sizes three and four, one of the workers does almost all the work (extremely large $P_{1}$ ). Due to the unbalanced workload, this model has very limited applicability at group tasks. A better approach when the group size is known is dividing jobs before studying how individuals learn their assigned tasks (similar to the product learning curve for $n$ separate operations in [16]). Once this is done, an aggregation of a group product becomes possible. 


\subsection{2. $C W L C$ (Eq. 6)}

A bivariate model by Camm and Womer [5] originally optimizes the group size for each repetition. The present CWLC model, however, assumes a fixed group size over repetitions. CWLC has three parameters, $B$ (which corresponds to $P_{1,1}$ in AWLC) and $g$ and $\gamma$ describing the contributions of group size and repetition. A $g=1.3$ in Table 1 indicates that adding a worker to a group has a diminishing return. Therefore, the CWLC is suitable when a task is divisible into components, and the number of workers performing it is a decision variable.

\subsubsection{PJLC (Eq. 7)}

This model is similar to the CWLC, but with the modifications, which are: (1) the group size parameter $\alpha$ corresponds to $1 / g$ in Eq. (6), and (2) the dependent variable is time per unit, and, consequently, $x$ and $n$ are raised to negative parameters. The parameter $y_{1}$ corresponds to $B$ in CWLC and $P_{1,1}$ in AWLC. This model is equally applicable as CWLC; however, its group size and learning parameters help better compare their effects. Both models fitted the data well, with the PJLC being slightly better.

\subsubsection{GJLC (Eq. 8)}

This model combines learning by doing (AWLC) and knowledge transfers (2PE) between each pair of individuals. Having more parameters, GJLC, however, performs better than AWLC only for groups of size three, and for others equally (Table 1). It is worth noting that knowledge transfer needs at least two workers and does not apply to those who worked alone. For groups of size four, the values of parameter $\delta$ are very high, indicating a negligible knowledge transfer. That simple AWLC performed equally for groups of two and four individuals indicates that workers in groups were homogeneous learners. Delaying knowledge transfer enables the GJLC to capture the group size effect, especially when a large group has, relatively, low production rate at the early stage of learning. The GJLC would have shown better results if the experiment went significantly beyond four repetitions. The GJLC emphasizes the importance of knowledge sharing and what may affect it.

\subsubsection{NBLC, MVLC-H and MVLC-E (Eqs. 9-11)}

The remaining three learning curve models represent productivity of a worker in a group. They focus on the maximum productivity, $k_{i}$, of (an average) worker, rather than the initial one (as in AWLC and GJLC). This feature could help in predicting performance in future production periods. The NBLC and MVLC-H are of a hyperbolic form and perform equally. The MVLC-E performs better than the two above, and as well as 2PE. For each of the three models, the learning parameter values $k_{i}$ and $\tau_{i}$ are, probably, too optimistic. The knowledge transfer parameter, $\theta_{i}$, represents an individual's capability to learn via transfer, and it is lower for larger groups (Table 1) which is realistic. It measures an individual's willingness/ability to absorb knowledge, similar to the one in the model of Glock and Jaber [6]; however, it was omitted from GJLC (Eq. 8) for simplicity.

The models of Méndez-Vázquez [7] are the only models that differentiate the effect of process loss from that of knowledge transfer in a group. The process loss parameter $L_{n}$ assumes a fixed value, which increases with group size. In general, the negative effect 
of process loss is compensated with greater knowledge transfer than for NBLC. Considering both process loss and knowledge transfer would help production managers in better decision making.

The above discussion implies that the Logistic model, of the models that use the cumulative production (repetition) as the proxy of knowledge, is the best in estimating and characterizing group learning in production processes. The S-shaped function, therefore, is better in capturing group size effect over time (Figure 1a) and in reflecting group learning challenges. The CWLC and the PJLC group learning curves are good alternative models when considering the number of workers. They allow one to simultaneously see the effects of group size and repetition over a learning session. The NBLC, MVLC-H, MVLC-E and GJLC models captured knowledge transfer, however, the latter provided unrealistic values. One reason for this is that GJLC is an aggregate model and considers worker-specific values which tend to be unequal for a better fit. Another reason is that the number of repetitions was significantly low for the learning data used.

\section{Conclusions and future lines of research}

The eleven learning curve models studied in this paper, which are of different mathematical forms, show how performance improves as a function of cumulative production, and what are the variables and parameters that affect it. The models were fitted to data from an experimental study where groups of one to four workers, assembled the case product four times. The groups were self-directed and suitable for estimating their learning process. The results showed that, in general, the more parameters in the model, e.g., Logistic and Plateau, the better the fit. A more detailed evaluation of the models suggests that the Logistic model and the two specific group learning models (Camm and Womer [5]; Peltokorpi and Jaber [8]) describe the group learning process and the group size effect the best. These models are most promising for predicting and analyzing group learning in production environments.

\subsection{Future lines of research}

A few studies (e.g., Glock and Jaber [6]; Nembhard and Bentefouet [23]; MéndezVázquez [7]) have incorporated the effect of knowledge transfer on group learning model. Modeling knowledge transfer in worker groups at a shared production task remains an open question for future research. Tacit knowledge transfer (learning from others) can occur in many ways. Letmathe et al. [24] studied how observation and imitation affect individual performance and learning at a production task. How learning through knowledge transfer occurs among group members has not been empirically proven in a production environment. For this reason, the current models are simplified and do not capture knowledge transfer in a way that contributes to decision making in a production setting. Many industrial jobs are performed in groups of two or three workers. In such groups, communication (both verbal and non-verbal) and observation would enhance knowledge transfer and learning and are worthy of investigating, which could include workers at different stages of career and levels of skills.

This study analyzed performance (operation time and productivity) with a strong focus on the application of knowledge transfer, which is the most pragmatic way to study group learning [25]. Further study should systematically analyze other group learning 
outcomes as well, and how various analytical models would estimate them. This includes a specialization, when individuals perform a narrower range of tasks over time [26], as group members tend to perform those tasks they can do most effectively [18]. The authors will address this aspect in future research. Also, product quality [27] is worthy of investigating and modeling group learning. Alongside with operative outcomes, one could model how worker related outcomes, such as work motivation and satisfaction, are affected by group learning (see, e.g., [28]). The number of workers also affects problemsolving capabilities and work ergonomics, which are vital for efficient production, and should, therefore, be modelled.

Future research will consider learning models for groups using analogies from other research fields and natural phenomena, such as the spread of infections [29]. Only a few studies have developed group learning curve models, as researchers traditionally have focused on individual and organizational learning models. Models associated with group learning are, mainly, conceptual, and research should take steps towards mathematical learning curve expressions that would benefit industrial decision making. On the one hand, this requires production history data on the performance of worker groups, preferably with different sizes, over consecutive repetitions. On another note, group learning data can also be gathered through controlled experiments. Future experimental research should consider a wide variety of group sizes and a large number of repetitions. Existing and new models can be fitted to new group learning data to determine which one describes its environment the best while evaluating the usability of the models in production decision making.

\section{Acknowledgement}

J. Peltokorpi thanks the Finnish Work Environment Fund (\#190242) for supporting his research. M.Y. Jaber thanks the Natural Sciences and Engineering Research Council of Canada (NSERC) for supporting his research.

\section{References}

[1] P.M. Pumilia-Gnarini, E. Favaron, E. Pacetti, J. Bishop and L. Guerra, Handbook of Research on Didactic Strategies and Technologies for Education: Incorporating Advancements, (2 volumes), IGI Global, Hershey, 2013.

[2] L.E. Yelle, The learning curve: historical review and comprehensive survey. Decision Sciences, vol. 10, 1979, pp. 302-328.

[3] M.Y. Jaber, (Ed.), Learning Curves: Theory, Models, and Applications, CRC Press (Taylor and Francis Group), FL, Boca Raton, 2011.

[4] T.P. Wright, Factors affecting the cost of airplanes, Journal of the Aeronautical Sciences, vol. 3, 1936, pp. $122-128$.

[5] J.D. Camm and N.K. Womer, Resource allocation in the crew assembly process, International Journal of Production Research, vol. 25, 1987, pp. 17-30.

[6] C.H. Glock and M.Y. Jaber, A group learning curve model with and without worker turnover, Journal of Modelling in Management, vol. 9, 2014, pp. 179-199.

[7] Y.M. Méndez-Vázquez, The Team Size Paradox: Knowledge Transfer and Process Loss Effects on Team Formation, 2019.

[8] J. Peltokorpi and M.Y. Jaber, A group learning curve model with motor, cognitive and waste elements, (unpublished).

[9] E.H. Grosse, C.H. Glock and S. Müller, Production economics and the learning curve: a meta-analysis, International Journal of Production Economics, vol. 170, 2015, pp. 401-412. 
[10] P. De Luca and M.C. Rubio, The curve of knowledge transfer: a theoretical model, Business Process Management Journal, vol. 25, 2019, pp. 10-26.

[11] J. Peltokorpi and E. Niemi, Effects of group size and learning on manual assembly performance: an experimental study, International Journal of Production Research, vol. 57, 2019, pp. 452-469.

[12] J.R. De Jong, The effects of increasing skill on cycle time and its consequences for time standards, Ergonomics, vol. 1, 1957, pp. 51-60.

[13] J.E. Mazur and R. Hastie, Learning as accumulation: a reexamination of the learning curve, Psychological Bulletin, vol. 85, 1978. pp. 1256-1274.

[14] R.H. Hou, S.Y. Kuo and Y.P. Chang, Applying various learning curves to hyper-geometric distribution software reliability growth model. In: Proceedings of IEEE International Symposium on Software Reliability Engineering, 1994, pp. 8-17.

[15] W. Hoppitt and K.N. Laland, Social Learning: An Introduction to Mechanisms, Methods, and Models, Princeton University Press, New Jersey, 2013.

[16] L.E. Yelle, Estimating learning curves for potential products, Industrial Marketing Management, vol. 5, 1976, pp. 147-154.

[17] N.K. Womer and T.R. Gulledge Jr, A dynamic cost function for an airframe production program, Engineering Costs and Production Economics, vol. 7, 1983, pp. 213-227.

[18] I.D. Steiner, Group Process and Productivity, Academic Press, New York, 1972.

[19] A.B. Badiru, Project Management in Manufacturing and High Technology Operations, $2^{\text {nd }}$ ed, John Wiley \& Sons, New York, 1996.

[20] J. Peltokorpi and E. Niemi, Analysis of the effects of group size and learning on manual assembly performance, Procedia Manufacturing, vol. 39, 2019, pp. 964-973.

[21] H.J. Leavitt, Some effects of certain communication patterns on group performance, The Journal of Abnormal and Social Psychology, vol. 46, 1951, pp. 38-50.

[22] L. Argote, C.A. Insko, N. Yovetich and A.A Romero, Group learning curves: the effects of turnover and task complexity on group performance 1, Journal of Applied Social Psychology, vol. 25, 1995, pp. 512 529.

[23] D.A. Nembhard and F. Bentefouet, Selection, grouping, and assignment policies with learning-by-doing and knowledge transfer, Computers \& Industrial Engineering, vol. 79, 2015, pp. 175-187.

[24] P. Letmathe, M. Schweitzer and M. Zielinski, How to learn new tasks: shop floor performance effects of knowledge transfer and performance feedback, Journal of Operations Management, vol. 30, 2012, pp. 221-236.

[25] C.W. Wiese and C.S. Burke, Understanding team learning dynamics over time, Frontiers in Psychology, vol. 10, 2019, 1417.

[26] M.A. Schilling, P. Vidal, R.E. Ployhart and A. Marangoni, Learning by doing something else: variation, relatedness, and the learning curve, Management Science, vol. 49, 2003, pp. 39-56.

[27] M.Y. Jaber and A.L. Guiffrida, Learning curves for processes generating defects requiring reworks, European Journal of Operational Research, vol. 159, 2004, pp. 663-672.

[28] S. Mat, K. Case, S. Mohamaddan and Y.M. Goh, A study of motivation and learning in Malaysian manufacturing industry, Production \& Manufacturing Research, vol. 5, 2017, pp. 284-305.

[29] H.S. Rodrigues and M.J. Fonseca, Can information be spread as a virus? Viral marketing as epidemiological model, Mathematical Methods in the Applied Sciences, vol. 39, 2016, pp. 4780-4786. 